\title{
Condicionamento litoestrutural e diferenciação dos vales do Planalto Vulcânico Sul-rio-grandense a partir de parâmetros morfométricos da drenagem
}

\author{
Michael V. de SORDI, Julio C. PAISANI \& Josielle S. PEREIRA
}

Núcleo de Estudos PaleoAmbientais, Universidade Estadual do Oeste do Paraná, Campus Francisco Beltrão. Av. Maringá, 1.200, CEP 85605-010, Francisco Beltrão, PR, Brasil (michael.sordi@gmail.com, juliopaisani@hotmail.com, josy.samara@hotmail.com).

\begin{abstract}
Resumo. Em escala regional, o estudo da morfologia dos vales e sua evolução de longotermo frente a ação de diferentes condicionantes ativos e passivos ainda é questão aberta no Planalto Vulcânico Sul-Rio-Grandense (PVSRG). Assim, no presente estudo foram empregados parâmetros morfométricos do gradiente dos canais, a identificação de knickpoints e $0 \quad k_{s}(n)$ (índice de inclinação normalizado) visando compreender as diferenças na morfologia e dinâmica erosiva de longo-termo nessa área. Os resultados mostram que, rios drenando litotipos ácidos e intermediários, das fácies Chapecó e Caxias apresentam gradiente acima de 0,013 e maior densidade de knickpoints (mais de 0,05 knickpoints por km de drenagem). Da mesma forma, os valores mais elevados de $\mathrm{k}_{\mathrm{s}}(\mathrm{n})$, acima de 17,9, ocorrem em vales sobre rochas ácidas. Por sua vez, vales instalados sobre rochas básicas e intermediárias, das fácies Esmeralda, Várzea do Cedro, Gramado, Paranapanema e Alegrete são mais planos, com gradiente abaixo de 0,010 e menos de 0,03 knickpoints por $\mathrm{km}$ de drenagem, refletindo em valores médios de $\mathrm{k}_{\mathrm{s}}(\mathrm{l})$ inferiores a 11,9. Portanto, a composição litológica das diferentes fácies é determinante para diferenciar os vales e condicionar a evolução dos cursos d'água no PVSRG.
\end{abstract}

Palavras-chave. Morfologia dos vales, Formação Serra Geral, erosão diferencial, índice $\mathrm{k}_{\mathrm{s}}\left(\mathrm{n}_{n}\right)$.

\begin{abstract}
LITHO-STRUCTURAL CONSTRAINTS AND VALLEY DIFFERENCIATION WITHIN SUL-RIOGRANDENSE VOLCANIC PLATEAU FROM DRAINAGE MORPHOMETRIC PARAMETERS. At regional scale, valley morphology study and its long-term evolution due to the action of different active and passive constraints is still an open question within Sul-Rio-Grandense volcanic plateau (PVSRG in Portuguese). Therefore, in the present study, morphometric parameters of river gradient knickpoint identification and $\mathrm{k}_{\mathrm{s}} \mathrm{S}_{\mathrm{n}}$ ) (normalized steepness index) were applied in order to identify differences in morphology and long-term erosive dynamics within overmentioned area. Results have shown that rivers draining acid and intermediary rocks from Chapecó and Caxias facies present gradient over 0,013 and higher knickpoint density (more than 0,05 knickpoints per length of drainage $(\mathrm{km})$ ). Equally, higher $\mathrm{k}_{\mathrm{s}}(\mathrm{n})$ values, above 17,9 occur on valleys over acid rocks. On the other hand, valleys installed on basic and intermediary rocks, from Esmeralda, Várzea do Cedro, Gramado, Paranapanema and Alegrete facies are flatter, with gradients below 0,010 and less than 0,03 knickpoints per drainage kilometer reflecting on average $k_{s}\left(l_{n}\right)$ values below 11,9 . Therefore, lithology composition is key to differentiate valleys and to restrain river evolution within PVSRG. Keywords. valley morphology, Serra Geral Formation, differential erosion, $\mathrm{k}_{\mathrm{s}}\left(\mathrm{n}_{\mathrm{n}}\right)$ index.
\end{abstract}




\section{Introdução}

A morfologia da paisagem regional, isto é, as formas de relevo, constituem testemunhos da ação de longo prazo dos agentes erosivodenudacionais sobre a superfície terrestre em conjunto com os agentes endógenos, que operam no interior da crosta (Molnar \& England, 1990; Summerfield, 1991; Bishop, 2007). Em áreas estáveis tectonicamente, a intensidade desses processos e, portanto, a configuração do relevo depende basicamente do balanço entre os agentes externos, ativos, e a resistência do embasamento à alteração - os agentes internos, passivos (Bishop, 2007; Rabassa, 2010; Gallen, 2018). Estudos recentes mostram que, enquanto rochas mais resistentes mantêm formas de relevo muito antigas, sob litotipos mais friáveis, a ação combinada dos agentes internos e externos remove quantidades expressivas de material e contribui para transformar as paisagens ao longo do tempo, sobretudo no período mais recente (Quaternário), mesmo em contextos considerados como tectonicamente estáveis (Bierman \& Caffee, 2001; Barreto et al., 2013; Couto et al., 2018; Sordi et al., 2018).

A compreensão da evolução do relevo passa pelo reconhecimento dos condicionantes geológico-geomorfológicos e da dinâmica evolutiva de longo-prazo da área em questão e de suas vizinhanças. Enquanto técnicas tradicionais de mapeamento geológico e geomorfológico consomem grande quantidade de tempo e recursos, o uso de parâmetros morfométricos como indicadores do controle litoestrutural na evolução do relevo de longo prazo das paisagens surge como uma alternativa rápida, eficiente e de baixo custo, mas que também permite uma leitura precisa de tais condicionantes na evolução da morfologia da paisagem (Howard, 1994; Wobus et al., 2006; Kirby \& Whipple, 2012; Lima, 2012; Lague 2014; Mudd, 2017; Xue et al., 2017; Whipple et al., 2017; Forte \& Whipple, 2018).

Por muito tempo, vigorou no Brasil a perspectiva de uniformidade das unidades litológicas vulcânicas no Brasil e foi comum a imagem de uma paisagem monótona nas áreas planálticas meridionais, compostas por extensas superfícies aplainadas (Ab'Saber,
1969). Regionalmente, o Planalto Vulcânico Sul-Rio-Grandense (PVSRG) foi descrito e compartimentado por diferentes autores ao longo dos anos (Leinz, 1949; Almeida, 1956; Ross, 1985; Justus et al., 1986; Dantas et al., 2010; Robaina et al., 2016). Recentemente, cita-se também estudos em escala de bacia hidrográfica (Robaina et al., 2010) e a nova delimitação proposta para o Planalto das Araucárias (Paisani et al., 2019), onde se reconhecem importantes e expressivas superfícies geomórficas (Biffi \& Paisani, 2019).

Assim, ainda que uma série de estudos de compartimentação e análise geomorfológica tenham sido realizados, nenhum teve como foco os condicionantes litológicos na diferenciação da morfologia dos vales na área do PVSRG, questão ainda em aberto. Desta forma, o presente estudo buscou identificar condicionantes litológicos na diferenciação da morfologia dos vales na área do PVSRG. Para isso, utilizou-se parâmetros morfométricos da rede hidrográfica: o gradiente, a identificação de knickpoints e o índice de inclinação normalizado $\mathrm{k}_{\mathrm{s}}(\mathrm{n})$.

\section{Materiais e métodos}

\subsection{Cenário geográfico}

O recorte utilizado como referência no presente estudo é o PVSRG (Fig. 1). As rochas vulcânicas da Formação Serra Geral afloram em uma área de $136.000 \mathrm{~km}^{2}$ no norte e oeste do Rio Grande do Sul. Tal formação pertence a Bacia Sedimentar do Paraná (BSP), e é composta por rochas vulcânicas datadas do Cretáceo Inferior (Comin-Chiaramonti et al., 2010, Polo \& Janasi, 2014; Besser et al., 2018; Rossetti et al., 2018) (Fig. 1). Pesquisas mais recentes sugerem que a Formação Serra Geral na área de estudo é constituída pela sobreposição das seguintes sequências de fácies: Esmeralda: derrames basálticos, microgranulares (Peate et al., 1992; Rossetti et al., 2018); Chapecó: derrames ácidos contendo traquitos, dacitos, riodacitos a riolitos (Nardy et al., 2008; Janasi et al., 2011) datados por Ar-Ar em 131,1 $\pm 0,2 \mathrm{Ma}$ (Wildner et al., 2006); Várzea do Cedro: derrames vitrofíricos pretos, texturas de fluxo e autobrechas frequentes, fino fraturamento perlítico (Wildner et al., 2006); 
Caxias: derrames de composição intermediária a ácida, riodacitos a riolitos, 132,3 $\pm 0,5$ Ar-Ar (Wildner et al., 2006; Nardy et al., 2008); Gramado: basaltos e em menor proporção, andesitos (Peate et al., 1992), datados por Ar-Ar em 132,4 1,4 Ma (Wildner et al., 2006); Paranapanema: derrames basálticos contendo horizontes vesiculares espessos, localmente preenchidos por quartzo (ametista) (Peate et al., 1992) datadas em 136,2 22 Ar-Ar (Wildner et al, 2006); Alegrete: derrames básicos a intermediários, variando de basaltos, andesitos-basálticos e andesitos (Martins et al., 2011), datados por Ar-Ar em 127,6 $\pm 1,2 \mathrm{Ma}$ (Wildner et al., 2006). Os derrames vulcânicos cessaram antes do Aptiano ( $\geq 125 \mathrm{Ma}$ ) (Asmus, 1975; Turner et al., 1994).

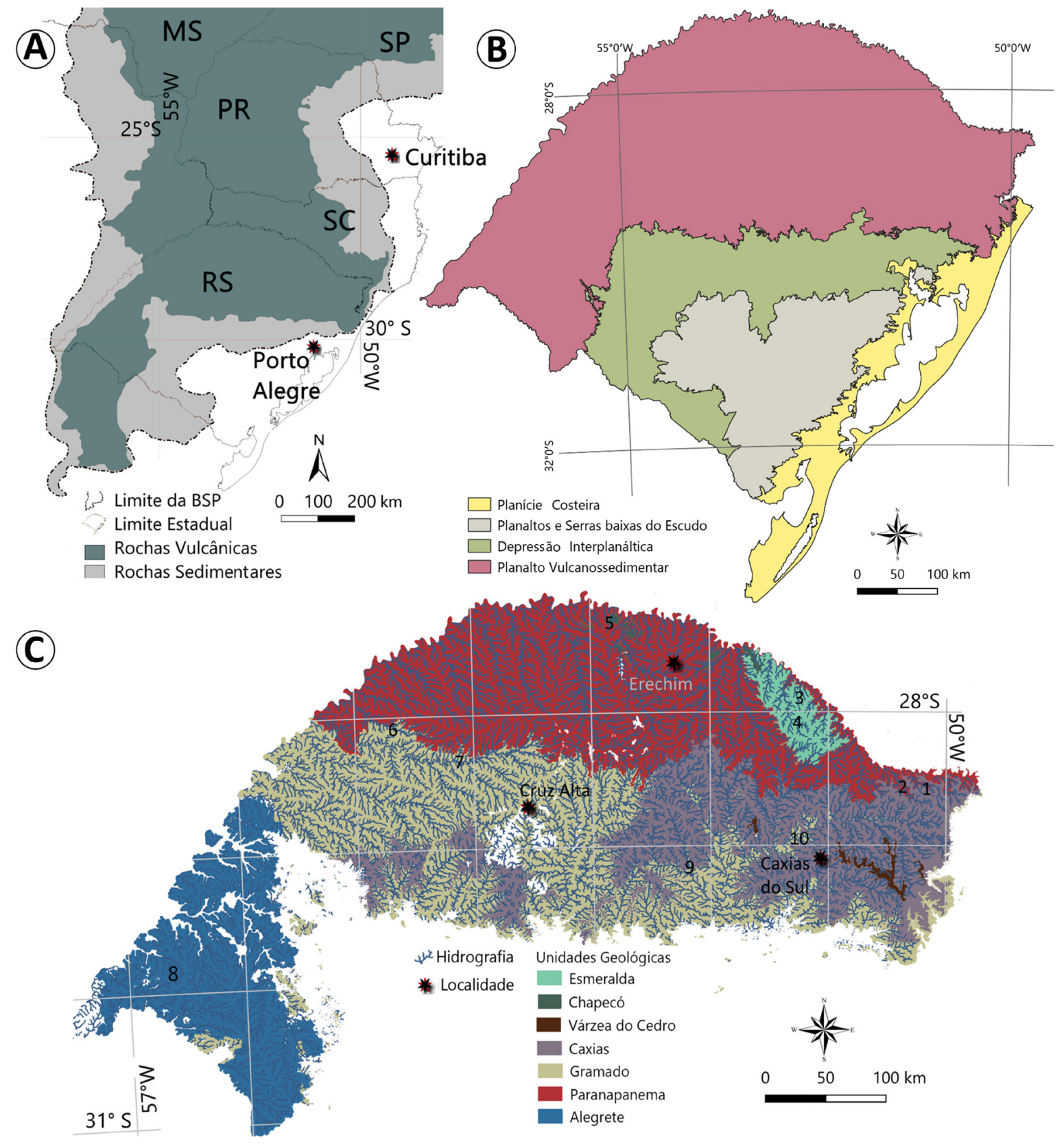

Figura 1. Contexto regional da Bacia Sedimentar no Sul do Brasil (BSP) (A). MS - Mato Grosso do Sul, PR - Paraná, SC - Santa Catarina, RS - Rio Grande do Sul Grandes Compartimentos de relevo no Rio Grande do Sul (B). Na parte inferior: localização das unidades geológicas do PVSRG no norte/oeste do estado do Rio Grande do Sul (C) elaborado a partir dos dados de Wildner et al. 2006. Os números se referem aos cursos d'água cujos perfis topográficos estão representados na Figura 03.

Figure 1. Regional context of Parana Sedimentary Basin in southern Brazil (BSP) (A).MS - Mato Grosso do Sul, PR - Paraná, SC - Santa Catarina, RS - Rio Grande do Sul. Major relief compartments in Rio Grande do Sul (B). On the lower side: geological units location within PVSRG in northern/western Rio Grande do Sul (C), elaborated from Wildner et al. 2006. The numbers refer to river which longitudinal profiles are represented on Figure 03. 
O clima no Rio Grande do Sul enquadrase como temperado úmido (Moreno, 1961). Segundo Rossato (2011) se distinguem na área de afloramento das rochas vulcânicas três tipos climáticos: i) subtropical pouco úmido, com chuvas entre 1.200 e $1.500 \mathrm{~mm}$ anuais e temperaturas médias entre $17-20^{\circ} \mathrm{C}$, no sudoeste do Estado; ii) subtropical medianamente úmido a úmido, com médias anuais de precipitação entre 1.500-1.800 mm e temperaturas médias anuais entre $17-20^{\circ} \mathrm{C}$, na faixa centro-oeste; iii) subtropical muito úmido, que ocorre no norte do estado, com precipitações acima dos $1.700 \mathrm{~mm}$ anuais e temperaturas médias entre $17-20^{\circ} \mathrm{C}$ no noroeste e entre $14-17^{\circ} \mathrm{C}$ no nordeste.

O relevo gaúcho pode ser compartimentado a partir de quatro grandes domínios geomorfológicos: planícies costeiras, planalto vulcanossedimentar (que compreende o PVSRG), depressões interplanálticas e os planaltos e serras baixas do Escudo (Dantas et al., 2010) (Fig. 1). Segundo Robaina et al. (2016), no PVRSG predominam encostas de baixa a média declividade com bases amplas. Considerando a compartimentação de Justus et al. (1986), - PVSRG pode ser subdividido nas seguintes regiões geomorfológicas: Planalto da Campanha, Planalto dos Campos Gerais, Planalto das Missões e o Planalto dissecado do rio Uruguai (Fig. 2B).

Quanto a hidrografia, no PVSRG predominam rios de leito rochoso. Os rios que drenam o Planalto dissecado do rio Uruguai, o Planalto da Campanha e as drenagens das porções norte do Planalto dos Campos Gerais e do Planalto das Missões, compõem a bacia do Uruguai. Dos afluentes do Uruguai se destacam os rios, Ibicuí, ljuí, Santa Maria e Piratini (Fig. 2A). Ainda é possível diferenciar as bacias orientadas para leste entre aquelas da região hidrográfica do Rio Guaíba, dos quais destacam-se os rios Jacuí e Antas-Taquari, com suas nascentes no Planalto dos Campos Gerais e das Missões (Justus et al., 1986) (Fig. 2B) e região hidrográfica litorânea, cujo maior curso d'água é o rio Camaquã (Fig. 2A).

\subsection{Procedimentos metodológicos}

O presente estudo se baseia na aplicação de parâmetros morfométricos e da revisão bibliográfica para reconhecimento do atual nível de conhecimento sobre a evolução do relevo na área analisada e sua caracterização geológicogeomorfológica. A partir dos parâmetros morfométricos elaborou-se perfis longitudinais, mapas de gradiente com os knickpoints, e mapas de $k_{s}\left({ }_{n}\right)$.

Para obtenção dos parâmetros morfométricos utilizou-se dados do NASADEM (NASA JPL, 2020), acessados pelo ASF-DAAC com resolução espacial de $30 \mathrm{~m}$, reprojetados para $90 \mathrm{~m}$ devido a extensão da área de estudo. Os dados foram manipulados e os parâmetros calculados no Topotoolbox, versão 2.3 (Schwanghart \& Kuhn, 2010; Schwanghart \& Scherler 2014), que se trata de um software de análise topográfica e hidrográfica baseado no Matlab® e distribuído gratuitamente. A hidrografia foi extraída automaticamente a partir dos dados de radar supracitados, utilizando o Topotoolbox.

Mapas espacializando gradiente dos canais, knickpoints e os valores de $k_{s}(n)$ permitiram reconhecer a morfologia dos canais. A declividade ou gradiente do canal (S) é inversamente proporcional a área drenada por um rio (A), assim pode se obter valores de $\mathrm{k}_{\mathrm{s}}$ a partir da Lei de Flint (Flint, 1974) (equação 1):

$$
\mathrm{S}=\mathrm{k}_{\mathrm{s}} \mathrm{A}^{-\theta} \rightarrow \mathrm{k}_{\mathrm{s}}\left(\mathrm{n}_{\mathrm{n}}\right)=\mathrm{S} / \mathrm{A}^{-\theta}
$$

onde 'S' corresponde ao valor da declividade, 'A' à área a montante do trecho analisado e ' $\theta$ (m/n)' ao índice de concavidade (Howard, 1994; Snyder et al., 2000; Wobus et al., 2006; Kirby \& Whipple, 2012). Geralmente, o teta $(\theta-(\mathrm{m} / \mathrm{n}))$ de referência para rios de leito rochoso varia entre 0,4 e 0,6 (Kirby \& Whipple, 2001, Giletycz et al., 2015). No presente estudo determinou-se os valores de $\theta$ por meio do software Topotoolbox - 0,39 para os afluentes do rio Uruguai e 0,42 para os canais que drenam diretamente para o Atlântico. 

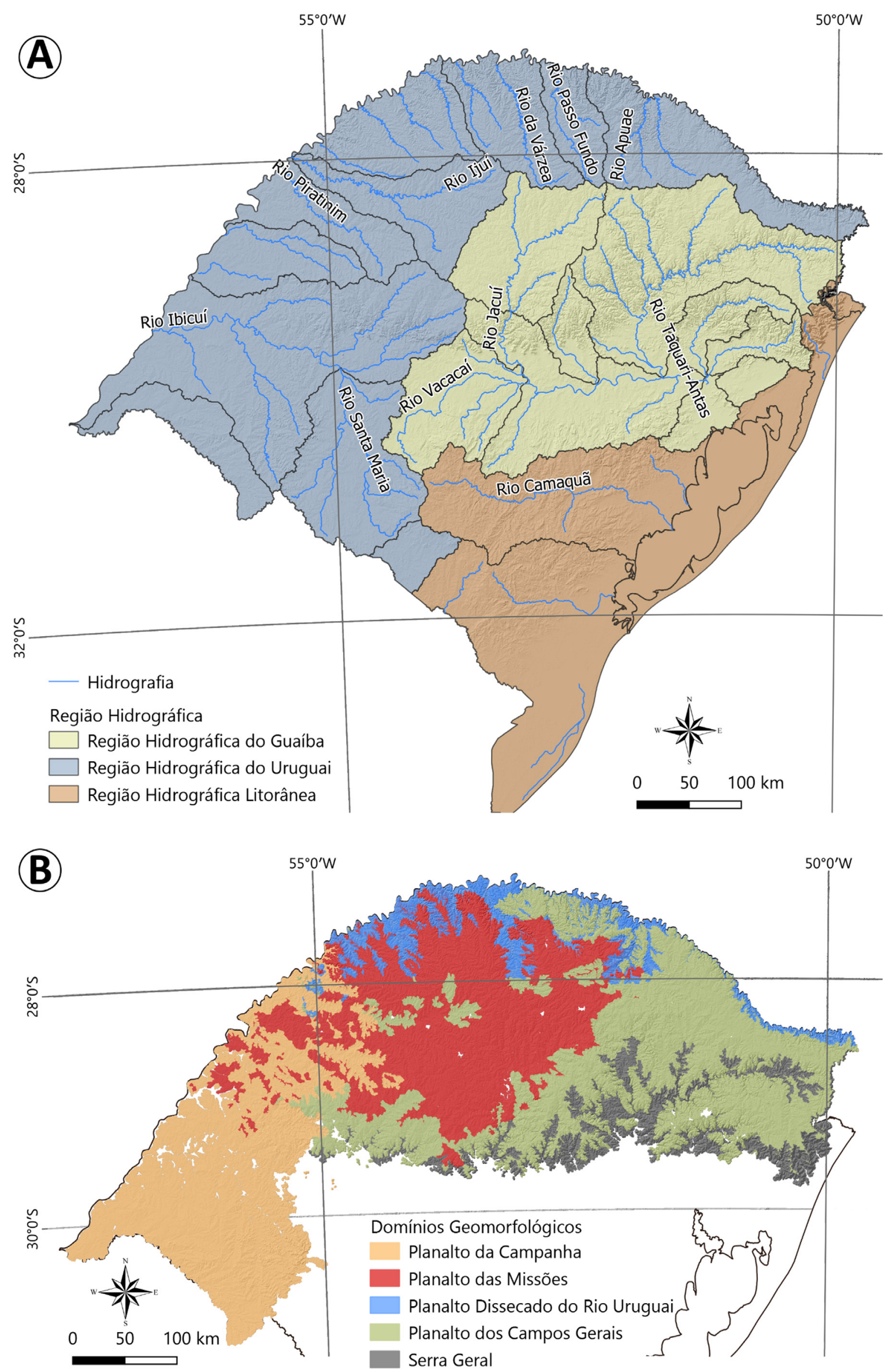

Figura 2. Regiões hidrográficas no Rio Grande do Sul (A) e Domínios Geomorfológicos delimitados por Justus et al. (1986) (B).

Figure 2. Hydrographic regions in Rio Grande do Sul (A) and Geomorphic domains defined by Justus et al. 1986 (B). 
O $\mathrm{k}_{\mathrm{s}}\left(\mathrm{n}_{\mathrm{n}}\right)$, ou Normalized Steepness index (índice de inclinação normalizado) (Wobus et al., 2006; Kirby \& Whipple, 2012), ajuda a comparar cursos d'água e identificar setores de declividade anômala, e inclusive, quebras de equilíbrio no perfil - que podem ou não constituir knickpoints, knickzonas ou quebras de relevo.

No presente estudo considera-se knickpoint como uma ruptura de declive no perfil longitudinal do canal, com desnível altimétrico maior que $30 \mathrm{~m}$ (vertical step na classificação de Lague, 2014). Na análise se considerou tanto knickpoints de origem tectônica quanto litoestrutural, inclusive aqueles no limite entre as litofácies. Para a obtenção dos knickpoints utilizou-se a função knickpointfinder do Topotoolbox, versão 2.3 (Schwanghart \& Kuhn, 2010; Schwanghart \& Scherler, 2014). A partir dessa função, os desníveis altimétricos são calculados a partir do deslocamento entre a elevação real e a elevação "ideal", definida a partir de uma curva de melhor ajuste em relação ao perfil longitudinal do canal.

\section{Resultados}

Para análise das características geomorfométricas da rede hidrográfica do PVSRG utilizou-se de perfis longitudinais, mapas de gradiente e o índice de inclinação normalizado $\left(k_{s}(n)\right)$, além dos knickpoints localizados ao longo dos canais.

Os perfis longitudinais de diferentes cursos d'água em toda a extensão do PVSRG mostram morfologias contrastantes entre os cursos d'água, onde se identificam canais e segmentos côncavos, retilíneos e convexos. Os segmentos convexos são mais comuns nas porções a montante dos canais. Tais áreas a montante geralmente se associam com as fácies Caxias e Chapecó (Rio dos Touros, Rio Cerquinha, Rio dos Índios, Fig. 3). Por vezes, também ocorrem perfis completamente côncavos, como é o caso do Arroio Touro Passo, que drena a Formação Alegrete (Fig. 3). Associada as Fácies Esmeralda, Gramado e Paranapanema também se notam morfologias - em perfil - côncavas (Rio ljuí, Rio Comandaí, Rio Pardo e Rio das Antas) (Fig. 3).

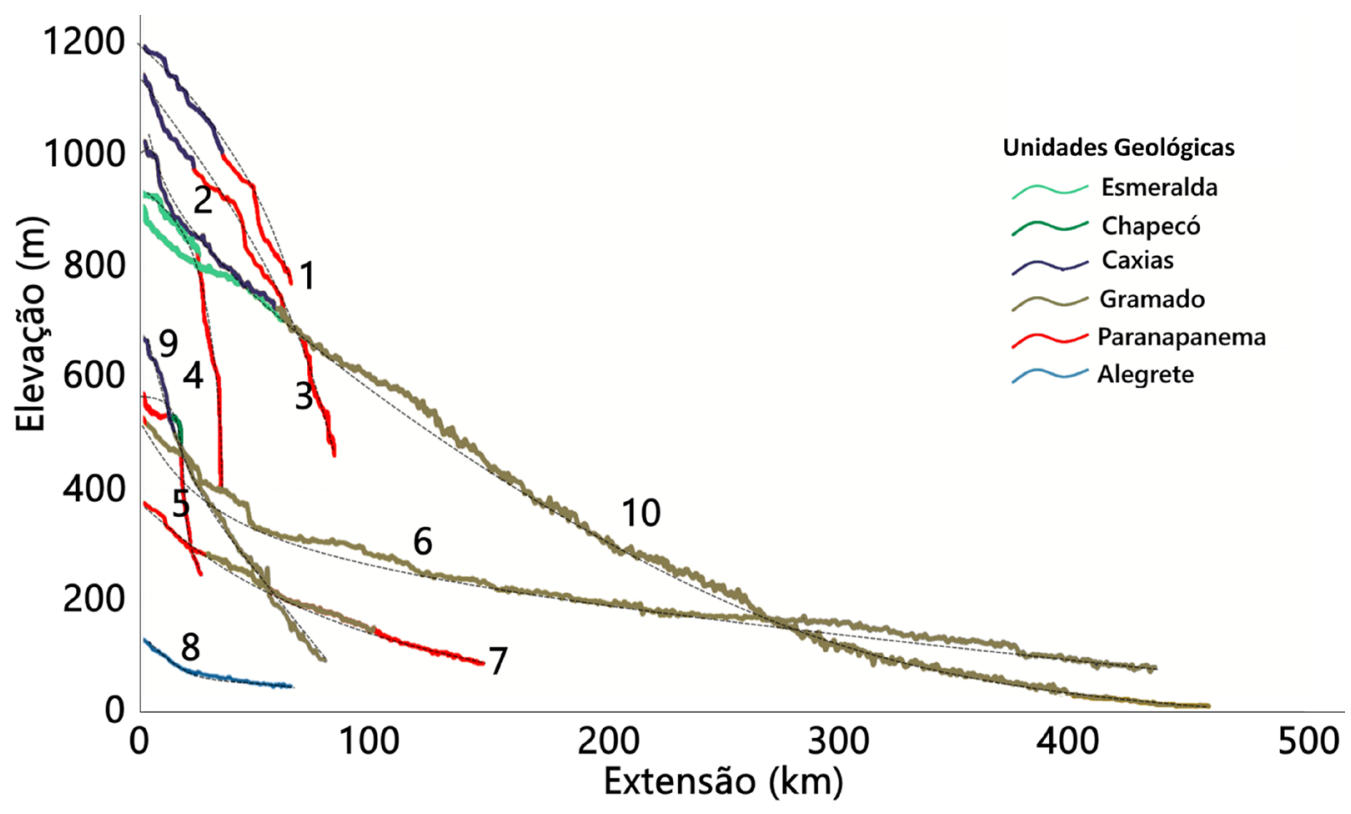

Figura 3. Perfis longitudinais de rios que drenam o PVSRG: 1: Rio Cerquinha; 2: Rio dos Touros; 3:Rio Bernardo José; 4: Rio do Frade; 5: Rio dos Índios; 6: Rio ljuí; 7:Rio Comandaí 8: Arroio Touro Passo; 9: Rio Pardo; 10: Rio das Antas

Figure 3. Longitudinal profiles of rivers draining PVSRG: 1: Rio Cerquinha; 2: Rio dos Touros; 3:Rio Bernardo José; 4: Rio do Frade; 5: Rio dos Índios; 6: Rio ljuí; 7:Rio Comandaí 8: Arroio Touro Passo; 9: Rio Pardo; 10: Rio das Antas 
No PVSRG foram perfilados 45.395 $\mathrm{km}$ de cursos d'água. Para melhor ilustrar as diferenças entre esses canais, dois parâmetros morfométricos foram mensurados nos cursos d'água que drenam as diferentes fácies geológicas do PVSRG: gradiente (Fig. 4) e índice de inclinação normalizado $\left(k_{s}(n)\right)$ (Fig. 5).

Se considerou 7 fácies geológicas pertencentes a Formação Serra Geral para as análises: Esmeralda, Chapecó, Várzea do Cedro, Caxias, Gramado, Paranapanema e Alegrete (Fig. 1). Na área de estudo, o gradiente médio dos canais é 0,009 com um desvio padrão nos valores de 0,012 . Ao analisar o mapa e a distribuição dos valores de gradiente, observa-se que os valores mais elevados ocorrem no quadrante leste, especialmente onde afloram as fácies Chapecó (média de 0,016; $n=74 ; 84,2$ km em extensão) e Caxias (média de 0,013; $n=644 ; 5.746$ km em extensão) (Fig. 4). Valores intermediários ocorrem nas fácies Esmeralda (média de 0,009; $\mathrm{n}=644 ; 1.011 \mathrm{~km}$ em extensão), Várzea do Cedro (média de 0,$01 ; n=58$ km em extensão) e Gramado (média de 0,01 n = 13.841; 18.914km em extensão) (Fig. 3), próximos a média regional no Planalto Vulcânico. Valores abaixo da média do PVSRG foram calculados para os canais que drenam a Fácies Paranapanema (média de 0,007; $n=9.607 ; 14.594$ km em extensão) (Fig. 4). Valores especialmente baixos ocorrem a oeste, nos afluentes do rio Uruguai, nas áreas onde o substrato corresponde a Fácies Alegrete - média de 0,003 ( $n=4.034 ; 5.746$ km em extensão), o que equivale a cerca de 5 vezes menos em relação as áreas onde afloram as rochas ácidas da Fácies Chapecó (Fig. 4).

Além disso foram localizados os desníveis topográficos nos canais que drenam o PVSRG, em um total de 994 knickpoints (Fig. 4) ao longo de cerca de $45.000 \mathrm{~km}$ de cursos d'água, o que resulta em uma densidade média de 0,02 knickpoints por km de drenagem. O desnível varia entre 30 m (mínimo mapeado) e 213 m (Fig. 4). 0 desnível médio é de $54 \mathrm{~m}$ e o desvio padrão nos valores é $82 \mathrm{~m}$.

O desnível médio é maior nos cursos d’água que drenam rochas das fácies Chapecó (86 m) e Esmeralda $(70 \mathrm{~m})$, mas esse valor elevado pode expressar a menor extensão areal dessas fácies na área de estudo. O valor médio dos desníveis é intermediário nas rochas das fácies Caxias (54m), Gramado (53m) e Paranapanema (53 m). Há menor desnível médio nos cursos d'água que drenam terrenos da fácies Alegrete (45 m). Só foi mapeado um knickpoint na Fácies Várzea do Cedro, com desnível de 116m.

Os knickpoints estão concentrados no leste da área de estudo, onde os cursos d'água drenam diretamente para o Atlântico, especialmente na bacia do rio Jacuí (Fig. 4). Se consideradas as fácies geológicas, a tendência é de maior ocorrência de knickpoints nas rochas das fácies Chapecó e Caxias (0,08 e 0,05 knickpoints por km de drenagem), bem acima da média regional de 0,02 knickpoints por km de drenagem. Identificase um grupo de concentração de knickpoints intermediário nas rochas das fácies Esmeralda e Gramado (0,03 knickpoints/km), Várzea do Cedro $(0,015$ knickpoints $/ \mathrm{km})$ e Paranapanema $(0,01$ knickpoints $/ \mathrm{km}$ ). Por fim, uma concentração muito baixa foi encontrada nos cursos d'água que drenam terrenos da Fácies Alegrete $(0,002$ knickpoints/km) (Fig. 4).

Por fim, foram mensurados os valores de $k_{s}(n)$ (Fig. 5). O valor médio de $k_{s}\left(l_{n}\right)$ nos rios que drenam o PVSRG é 12,7. A análise da Figura 5 mostra que os canais principais, em todo recorte de estudo, apresentam valores elevados de $k_{s}\left({ }_{n}\right)$, a exceção dos canais que drenam o sudoeste da área. Também se destaca o fato que valores anômalos são encontrados nos trechos médios e baixos dos canais, e não nas cabeceiras, onde se esperaria valores mais altos.

Quando se analisam os valores de $k_{s}\left({ }_{n}\right)$ nos cursos d'água nas diferentes fácies geológicas, novamente os valores mais elevados estão associados as fácies Caxias (19,0) e Chapecó $(17,9)$. Valores de $k_{s}\left({ }_{n}\right)$ elevados, de 16,5, também são encontrados nos canais que drenam as rochas da Fácies Gramado, acima da média do PVSRG. Os valores são intermediários, de 10,1 e 11,9 nas fácies Esmeralda e Várzea do Cedro, respectivamente. Para os cursos d'água com leito instalado sobre rochas da Fácies Paranapanema a média é menor, 8,6. Por fim, índices médios mais baixos, de 2,9, caracterizam os canais sobre a Fácies Alegrete. 


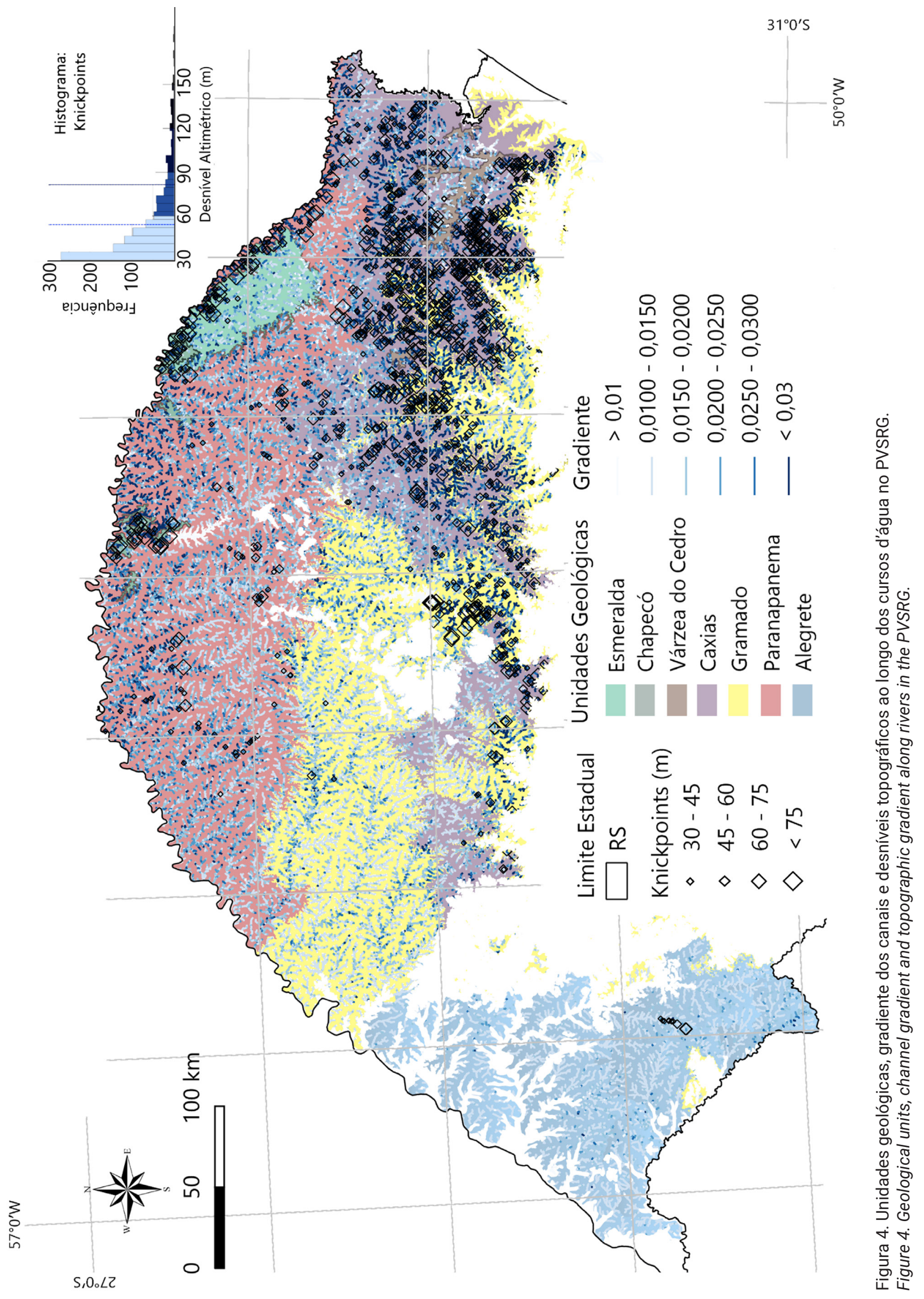




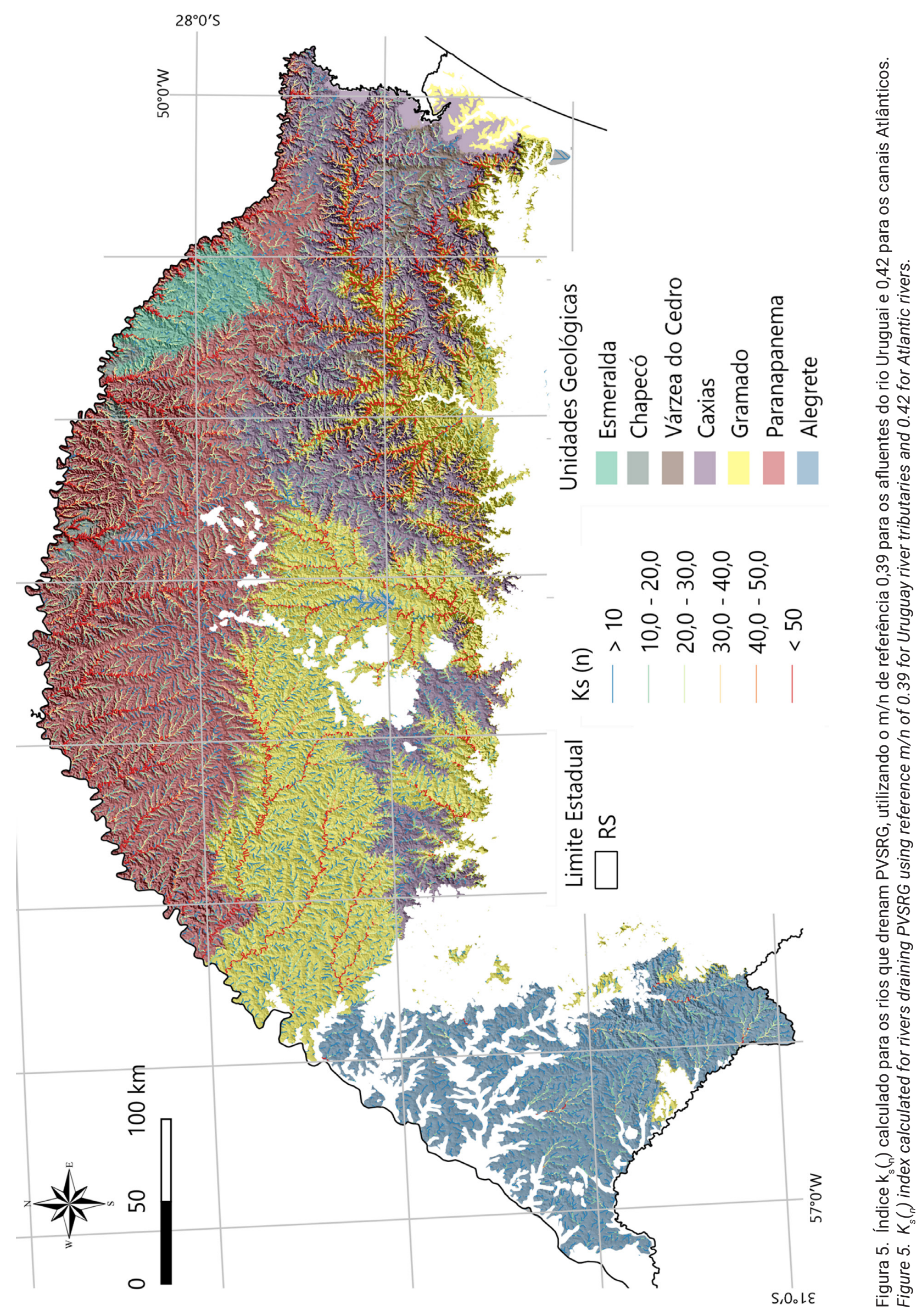




\section{Discussão dos resultados}

A análise da distribuição dos valores de gradiente, densidade de knickpoints e $k_{s}\left({ }_{n}\right)$ mostra que de fato há uma diferenciação entre os canais que drenam os diferentes litotipos que compõem a Formação Serra Geral no PVSRG. Ao plotar os valores de $\mathrm{k}_{\mathrm{s}}(\mathrm{n})$ e gradiente fica clara a diferenciação devido a influência dos litotipos (Fig. 6). Os vales mais íngremes, com maior gradiente e maiores valores de $k_{s}\left(l_{n}\right)$ ocorrem nas rochas das fácies Chapecó e Caxias, de composição intermediária a ácida (Peate et al., 1992; Janasi et al., 2011) ainda que existam importantes diferenças na composição e estruturação entre elas (Nardy et al., 2008). Índices de gradiente e $\mathrm{k}_{\mathrm{s}}(\mathrm{n})$ intermediário, do menor para o maior, ocorrem nas fácies Paranapanema, Esmeralda, Várzea do Cedro e Gramado (Fig. 5) (Peate et al., 1992; Rossetti et al., 2018). Tais fácies são compostas por membros básicos, principalmente basaltos e latitos. Vale ressaltar que, na área de afloramento da Fácies Gramado os índices morfométricos apresentam valores intermediários (Figs. 4, 5 e 6), mais próximos daqueles que ocorrem nas fácies Chapecó e
Caxias, resultado da sua composição básica a intermediária. Por fim, sobre a Fácies Alegrete, composta de basaltos a andesitos (Martins et al., 2011) os vales têm menor declividade e os valores de $\mathrm{k}_{\mathrm{s}}(\mathrm{n})$ e gradiente são muito menores (Fig. 6).

Assim, em grande medida, a diferenciação nesses índices é explicada pelas propriedades e composição dessas rochas. Além disso, em escala de maior detalhe, há uma diferenciação entre as rochas básicas com alto teor de TiO2 (Paranapanema) (Peate et al., 1992) (derrames toleíticos-transicionais) e rochas com baixo teor de $\mathrm{TiO}_{2}$ (Gramado, Esmeralda) (derrame toleítico) (Peate et al., 1992). Da mesma forma, indícios de elevada correlação entre os valores de $k_{s}\left({ }_{n}\right)$ e taxas de denudação (Xue et al., 2017) apontam para uma dinâmica erosiva diferenciada que, em longo-termo irá originar morfologias diferentes nos diferentes litotipos vulcânicos. No Planalto Catarinense, as taxas de denudação sobre as rochas vulcânicas básicas $\left(6,1 \pm 0,7 \mathrm{~m} / \mathrm{Ma}^{-1}\right.$, $\mathrm{n}=2$ ) são, em média, duas vezes maiores quando comparadas as taxas de denudação em rochas vulcânicas ácidas, do tipo Chapecó (3,3 \pm 0,4 m/ $\left.\mathrm{Ma}^{-1}\right)(\mathrm{n}=2)$ (Sordi et al., 2018).

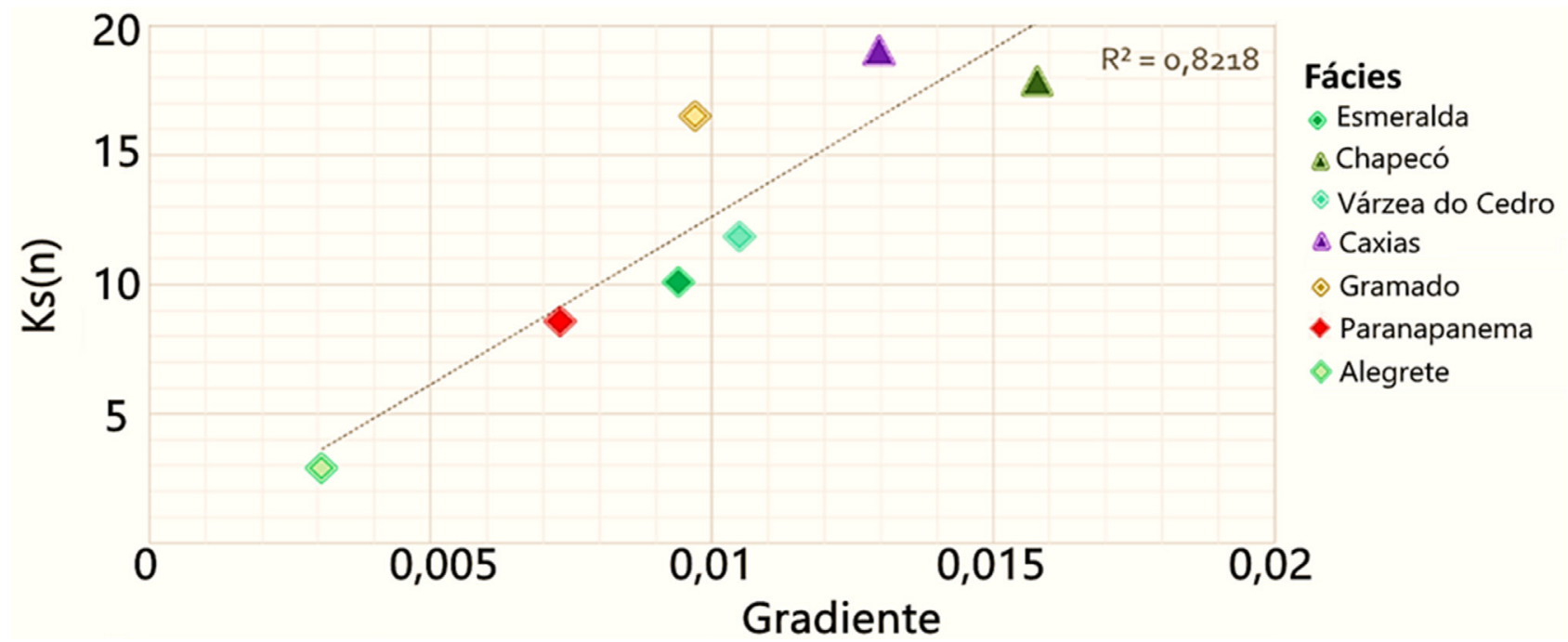

Figura 6. Variação dos parâmetros morfométricos dos vales no PVSRG $\left(\mathrm{k}_{\mathrm{s}}\left(\mathrm{n}_{\mathrm{n}}\right)\right.$ e gradiente) nas diferentes fácies que compõe a Formação Serra Geral.

Figure 6. Morphometric indexes variations within valleys in PVSRG $\left(k_{s}\left({ }_{n}\right)\right.$ and gradient) on the different facies which compound Serra Geral Formation. 
Tais resultados são reforçados pela densidade de knickpoints, maior nas fácies vulcânicas de composição intermediária a ácida (Chapecó e Caxias) que sobre as fácies de composição básica (Gramado, Esmeralda, Várzea do Cedro e Paranapanema), e especialmente nos vales drenando as rochas da Fácies Alegrete (Fig. 4). A diferença na densidade de rupturas de declive no interior das unidades mapeadas por Wildner et al. (2006) sinaliza a existência de importantes diferenças entre as fácies, já que os parâmetros aqui utilizados são bons indicativos de mudanças de composição e estruturação, além das anomalias estruturais (Brocard \& Van Der Beek, 2006).

Ainda que outros condicionantes sejam importantes na evolução da paisagem, como paleoclima, relevo prévio, cobertura superficial, tectônica, dentre outros (Mudd, 2017), o que fica claro é que as diferenças na composição dos diferentes derrames e fácies influencia na morfologia dos vales, mas em última análise, também das paisagens no PVSRG. Esclarecer as diferenças entre morfologias e eventuais associações com diferentes tipos de rochas é essencial para compreender a evolução de longotermo dos vales e as paisagens atuais sobre rochas vulcânicas.

\section{Considerações finais}

De modo generalizado se observa uma diferenciação principal entre rios com leito sobre rochas vulcânicas ácidas em relação aos rios que drenam rochas vulcânicas intermediária e básicas.

Sob rochas ácidas e intermediárias, das fácies Chapecó e Caxias, os vales são íngremes e apresentam elevado gradiente, acima de 0,013, e maior densidade de knickpoints, mais de 0,05 knickpoints por km de drenagem o que por sua vez se reflete em valores mais elevados de $k_{s}(n)$, acima de 17,9. Os vales instalados sobre rochas básicas e intermediárias, das fácies Gramado, Esmeralda, Várzea do Cedro e Paranapanema são menos íngremes, com menor gradiente, abaixo de 0,010, e menor quantidade de knickpoints: menos de 0,03 por km de drenagem. Isso se reflete em menores valores médios de $\mathrm{k}_{\mathrm{s}}\left(\mathrm{n}_{\mathrm{n}}\right)$, inferiores a
11,9. Na Fácies Alegrete ocorrem os menores valores de gradiente, $\mathrm{k}_{\mathrm{s}}(\mathrm{n})$ e a menor densidade de knickpoints.

Os dados aqui apresentados e interpretados evidenciam a importância da análise de parâmetros morfométricos em estudos da evolução e dinâmica dos rios de leito rochoso. No caso do PVSRG existe uma clara diferenciação entre os vales e as características litoestruturais das diferentes fácies que compõe a Formação Serra Geral deve ser considerada na compreensão dessas diferenças.

Agradecimentos. Os autores agradecem a CAPES (Coordenação de Aperfeiçoamento de Pessoal de Nível Superior) à bolsa de doutorado da terceira autora do manuscrito.

\section{Referências}

Ab'Saber, A.N. 1969. Participação das superfícies aplainadas nas paisagens do Rio Grande do Sul. Geomorfologia, 11: 1-17.

Almeida, F.F.M. 1956. O Planalto basáltico da Bacia do Paraná. Boletim Paulista de Geografia, 24: 3-34.

Asmus, H.E. 1975. Controle Estrutural da Deposição Mesozoica nas Bacias da Margem Continental Brasileira. Revista Brasileira de Geociências, 5 (3): 160-175.

Barreto, H.N., Varajão, C.A.C., Braucher, R., Bourlés, D. \& Salgado, A.A.R. 2013. Denudation rates of the Southern Espinhaço Range, Minas Gerais, Brazil, determined by in situ-produced cosmogenic beryllium-10. Geomorphology, 191: 1-13.

Besser, M. L., Vasconcellos, E.M.G. \& Nardy, A.J.R. 2018. Morphology and stratigraphy of Serra Geral silicic lava flows in the northern segment of the Torres Trough, Paraná Igneous Province. Brazilian Journal of Geology, 48(2): 201-219.

Bierman P.R. \& Caffee, M. 2001. Slow rates of rock surface erosion and sediment production across the Namib desert and escarpment, southern Africa. American Journal of Science, 301: $326-358$.

Bishop, P. 2007. Long-term landscape evolution: Linking tectonics and surface processes. 
Earth Surface Processes and Landforms, 32: 329-365.

Biffi, V.H.R. \& Paisani, J.C. 2019. Micromorfologia de colúvio-alúvios em paleovoçorocas colmatadas nas superfícies de cimeira de Pinhão/Guarapuava e Palmas/Caçador - sul do Brasil. Revista Brasileira de Geomorfologia, 20: 735-749.

Brocard, G.Y. \& Van Der Beek, P. 2006. Influence of incision rate, rock strength and bedload supply on bedrock river gradients and valleyflat widths: field-based evidence and calibrations from western Alpine rivers (SE France). In: Willett, S.D., Brandon, M.T. \& Fisher, D. (Eds). Tectonics, Climate and Landscape Evolution,). Geological Society of America Special Paper. Geological Society of America: Washington, DC, 101-126.

Comin-Chiaramonti, P., Riccomini, C., Slejko, F., De Min, A., Ruberti, E. \& Gomes, C.B. 2010. Cordierite-bearing lavas from Jaguarão, Southern Brazil: Petrological evidence for crustal melts during early rifting of Gondwana. Gondwana Research, 18: 514-527.

Couto, E.V., Santos, L.J.C., Sordi, M.V., Bourlés, D., Braucher, R. Salgado, A.A.R., Léanni L., Ferreira, J.H.D. \&Aster TEAM. 2018. Changes of the base levels in the Ivaí and Paraná Rivers confluence zone (Southern Brazil): Denudational reflexes in the evolution of the upstream drainage network. Zeitschrift Für Geomorphologie, 62 (1): 23-40.

Dantas, M.E., Viero, A.C. \& Silva, D.R.A. Da. 2010. Origem das paisagens. In: Viero, A.C. \& Silva, D.R.A. da. Geodiversidade do Estado do Rio Grande do Sul. Porto Alegre: CPRM, p. 35-50.

Flint, J.J. 1974. Stream gradient as a function of order, magnitude and discharge. Water Resources Research, 10(5): 969-973.

Forte, A.M. \& Whipple, K. 2018. Criteria and tools for determining drainage divide stability. Earth and Planetary Science Letters, 493, 102-117.

Giletycz, S., Loget, N., Chang, Cp. \& Mouthereau, F. 2015. Transient fluvial landscape and preservation of low-relief terrains in an emerging orogen: Example from Hengchun Peninsula, Taiwan. Geomorphology, 231: 169181.

Howard, A.D. 1994. A detachment-limited model of drainage basin evolution. Water Resources Research, 30: 2261-2285.

Justus, J.O., Machado, M.L.A. \& Franco, M.S.M. Geomorfologia. In: Projeto RADAMBRASIL, 33. Folha SH-22 - Porto Alegre e parte das folhas SH-21 - Uruguaiana e SI-22 - Lagoa Mirim. Rio de Janeiro: IBGE, 1986, p. 313-404.

Kirby, E. \& Whipple, K. 2001. Quantifying differential rock-uplift rates via stream profile analysis. Geology, 29: 415-418.

Kirby, E. \& Whipple, K.X. 2012. Expression of active tectonics in erosional landscapes. Journal of Structural Geology, 44, 54-75.

Lague, D. 2014. The stream power river incision model: evidence, theory and beyond. Earth Surf. Process. Landforms, 39: 38-61. https:// doi.org/10.1002/esp.3462

Leinz, V. 1949. Contribuição à geologia dos derrames basálticos do sul do Brasil. Geologia, Boletim CIII, n. 5, Faculdade de Filosofia, Ciências e Letras, USP, 61p.

Lima, A.G. 2009. Controle geológico e hidráulico na morfologia do perfil longitudinal em rio sobre rochas vulcânicas básicas da Formação Serra Geral no Estado do Paraná. Florianópolis, 240p. Tese de Doutorado, Programa de PósGraduação em Geografia, Universidade Federal de Santa Catarina.

Lima, A.G. 2012. Erosão fluvial sobre rochas vulcânicas: algumas inferências a partir de segmentos côncavos de perfis longitudinais. Revista Brasileira de Geociências, 42: 3441.

Lima, A.G. \& Binda, A.L. 2013. Lithologic and structural controls on fluvial knickzones in basalts of the Paraná Basin, Brazil. Journal of South American Earth Sciences, 48: 262-270. https://doi.org/10.1016/j.jsames.2013.10.004

Machado, J.L.F. 2005. Compartimentação espacial e arcabouço hidrogeológico do sistema aquífero Guarani no Rio Grande do Sul. São Leopoldo, 237p. Tese de Doutorado, PósGraduação em Geologia, Universidade do Vale do Rio do Sinos.

Martins, L.C., Wildner, W. \& Hartmann, L.A. 2011. Estratigrafia dos Derrames da Província Vulcânica Paraná na Região Oeste do Rio Grande do Sul, Brasil, com Base em Sondagem, Perfilagem Gamaespectrométrica e Geologia 
de Campo. Pesquisas em Geociências, 38(1); 15-27.

Molnar, P. \& England, P. 1990. Late Cenozoic uplift of mountain ranges and global climate change: chicken or egg? Nature, 346: 29-34.

Monteiro, C.A.F. 1968. Clima. In: IBGE. Geografia do Brasil, grande região sul. Rio de Janeiro, IBGE, 4(I): 114-166.

Moreno, J.A. 1961. Clima do Rio Grande do Sul. Porto Alegre: Secretaria do Estado do Rio Grande do Sul, 82p.

Mudd, S. M. 2017. Detection of transience in eroding landscapes. Earth Surf. Process. Landforms, 42: 24-41.

Nardy, A.J.R., Machado, F.B. \& Oliveira, M.A.F. As rochas vulcânicas mesozóicas ácidas da Bacia do Paraná: litoestratigrafia e considerações geoquímico-estratigráficas. Revista Brasileira de Geociências, 38(1): 178-195, 2008.

NASA JPL. 2020. NASADEM Merged DEM Global 1 arc second V001 [Data set]. NASA EOSDIS Land Processes DAAC. Accessed 2020-0708 from https://doi.org/10.5067/MEaSUREs/ NASADEM

Paisani, J.C., Santos, L.J.C., Goudard, G., Goulart, A.A. \& Biffi, V.H.R. 2019. Subtropical Araucárias Plateau. In: Salgado, A.A., Santos, L.J.C. \& Paisani, J.C. (Eds.), Physical geography of Brazil. Springer, Cham, 21-40.

Peate, D.W., Hawkeswort, C.J. \& Mantovani, M.S.M. 1992. Chemical stratigraphy of the Paraná lavas (South America): classification of magma types and their spatial distribution. Bulletin of Volcanology, 55:119-139

Polo, L.A. \& Janasi, V.A. 2014. Volcanic stratigraphy of intermediate to silicic rocks in Southern Paraná Magmatic Province, Brazil. Geologia USP Série Científica, 14 :

83-100.

Robaina, L.E.S., Trentin, R. Bazzan, T., Reckziegel, E.W., Verdum, R \& Nardin, D. 2010. Compartimentação Geomorfológica Da Bacia Hidrográfica Do Ibicuí, Rio Grande Do Sul, Brasil: Proposta De Classificação. Revista Brasileira de Geomorfologia, 11(2): 11-23.

Robaina, L.E.S., Trentin, R. \& Laurent. F. 2016. Compartimentação do estado do Rio Grande do Sul, Brasil, através do uso de geomorphons obtidos em Classificação topográfica automatizada. Revista Brasileira Geomorfologia, 17(2): 287-298.

Rossetti, L., Lima, E.F., Waichel, B.L., Hole, M.J., Simões, M.S. \& Scherer, C.M.S., 2018. Lithostratigraphy and volcanology of the Serra Geral Group, Paraná-Etendeka Igneous Province in Southern Brazil: Towards a formal stratigraphical framework. Journal of Volcanology and Geothermal Research, 355: 98-114.

Rossato, M.S. 2011. Os climas do Rio Grande do Sul: variabilidade, tendências e tipologia. Porto Alegre, 240 p. Tese de Doutorado, Programa de Pós-graduação em Geociências, Instituto de Geociências, Universidade Federal do Rio Grande do Sul.

Ross, J.L.S. 1985. Relevo brasileiro: uma nova proposta de classificação. Revista do Departamento de Geografia, 4:25-39.

Schwanghart, W. \& Scherler, D. 2014. TopoToolbox 2 - MATLAB-based software for topographic analysis and modeling in Earth surface sciences. Earth Surface Dynamics, 2: 1-7.

Schwanghart, W. \& Kuhn, N. J. 2010. TopoToolbox: a set of MATLAB functions for topographic analysis. Environmental Modelling \& Software, 25: 770-781.

Snyder, N.P., Whipple, K.X., Tucker, G.E. \& Merritts, D.J. 2000. Landscape response to tectonic forcing: DEM analysis of stream profiles in the Mendocino triple junction region, northern California. Geological Society of America Bulletin, 112: 1250-1263.

Sordi, M.V. Salgado, A.A.R., Paisani, J.C. \& Pagotto, D. 2017. Controle litoestrutural no desenvolvimento de vales na área do rebordo da Bacia do Paraná no Estado de Santa Catarina, Sul do Brasil. Revista Brasileira de Geomorfologia, 18(4): 671-687.

Sordi, M. V., Salgado, A. A. R., Siame, L., Bourlés, D., Paisani, J. C., Leanni, L., Braucher, R., Couto, E. V. 2018 \& ASTER TEAM. Implications of drainage rearrangement for passive margin escarpment evolution in southern Brazil. Geomorphology, 306: 155-169.

Summerfield, M. A. 1991. Global Geomorphology. John Wiley and Sons, New York, 537p.

Turner, S., Regelous, M., Kelley, S., Hawkeswort, 
C.J. \& Mantovani, M.S.M. 1994. Magmatism and continental break-up in the South Atlantic: high precision 40Ar/39Ar geochronology. Earth and Planetary Science Letters, 121:333-348.

Whipple, K.X., Forte, A.M., Dibiase R.A. Gasparini., N.M. \& Ouimet W. B. 2017. Timescales of landscape response to divide migration and drainage capture: Implications for the role of divide mobility in landscape evolution. Journal of Geophysical Research: Earth Surface,122: 248-273.

Wildner, W., Ramgrag, G. E., Lopes, R. C. \& Iglesias, C. M. F. 2006. Mapa Geológico do Estado do Rio Grande do Sul. Escala 1:750000. CPRM, Serviço Geológico do Brasil, Porto Alegre.

Wobus, C., Whipple, K.X., Kirby, E, Snyder, N., Johnson, J., Spyropolou, K., Crosby, B. \& Sheehan, D. 2006. Tectonics from topography: Procedures, promise, and pitfalls. Geological Society of America Special Papers, 398: 55-74.

Xue, L., Gani, N.D. \& Abdelsalam, M.G. 2017. Geomorphologic proxies for bedrock rivers: A case study from the Rwenzori Mountains, East African Rift system. Geomorphology, 285: 374398. 